\title{
LA IDENTIDAD INDÍGENA INTERPRETADA COMO UNA CATEGORÍA DE ANÁLISIS EN LOS ESTUDIOS DE POBLACIÓN
}

\author{
THE INDIGENOUS IDENTITY INTERPRETED AS A CATEGORY OF ANALYSIS IN POPULATION STUDIES
}

A IDENTIDADE INDÍGENA INTERPRETA COMO CATEGORIA DE ANÁLISE EM ESTUDOS DE POPULAÇÃO

\author{
Dante Choque-Caseres \\ University of Sydney. Sydney, Australia. \\ Centro de Estudios Interculturales e Indígenas. Santiago, Chile. \\ dchoquec@gmail.com \\ ORCID: 0000-0002-3300-8864
}

DOI: https://doi.org/10.22235/ech.v6iEspecial.1457
Recibido: $15 / 06 / 2017$
Aceptado: $30 / 08 / 2017$

\section{RESUMEN}

En Latinoamérica, a partir del reconocimiento de los Pueblos Indígenas, la identificación de brechas o disparidades entre la población Indígena y no Indígena ha emergido como un interés investigativo. Con este fin, capturar la identidad Indígena es clave para conducir algunos análisis. Sin embargo, los contextos sociales donde se (re)produce la identidad de las personas Indígenas han sido alterados significativamente. Dichos cambios son producidos por la asimilación o integración de las comunidades Indígenas a las culturas nacionales dominantes. En este contexto emergen limitaciones en el uso de esta categoría, puesto que la identidad Indígena tendría un componente político y legal relacionado con las necesidades de los gobiernos. Por lo tanto, una reflexión crítica sobre el uso de la identidad Indígena es necesaria en los enfoques epistemológicos y metodológicos de investigación.

Este artículo argumenta que la investigación sobre pueblos Indígenas debería evaluar el cómo es incluida la identidad Indígena, porque ésta es socialmente co-producida en la interacción con el Estado y sus instituciones. De esta forma, ella no necesariamente podría constituir una variable explicativa. A través del análisis de discursos acerca de personas y comunidades Indígenas Aymaras que han emergido en la frontera norte de Chile busco exponer las lógicas empleadas para definir la identidad. Se concluye, entonces, que paulatinamente el proceso de auto-identificación emerge en supuestos Indígenas constituidos y/o reforzados por las instituciones, los cuales deberían ser revisados desde un enfoque descolonizado para ser incluidas en investigaciones comparativas.

Palabras clave: Indígenas, Identidad, Censo.

\section{ABSTRACT}

In Latin America, based on the recognition of Indigenous Peoples, the identification of gaps or disparities between the Indigenous and non-Indigenous population has emerged as a new research interest. To this end, capturing Indigenous identity is key to conducting certain analyses. However, the social contexts where the identity of Indigenous persons are (re)produced has been significantly altered. These changes are generated by the assimilation or integration of Indigenous communities into dominant national cultures. Within this context, limitations emerge in the use of this category, since Indigenous identity has a political and legal component related to the needs of the government. Therefore, critical thought on the use of Indigenous identity is necessary in an epistemological and methodological approach to research.

This article argues that research about Indigenous Peoples should evaluate how Indigenous identity is included, for it is socially co-produced through the interaction of the State and its institutions. Thus, it would not necessarily constitute an explicative variable. By analyzing the discourse about Aymara Indigenous communities that has emerged in the northern border of Chile, this paper seeks to expose the logic used to define identity. Therefore, I conclude that the process of self-identification arises in supposed Indigenous people, built and/or reinforced by institutions, which should be reviewed from a decolonizing perspective and included in comparative research.

Keywords: Indigenous, Identity, Census.

\section{RESUMO}

Na América Latina, a partir do reconhecimento dos Povos Indígenas, a identificação de lacunas ou disparidades entre a população indígena e não-indígena surgiu como interesse de pesquisa. Com essa finalidade, resgatar a identidade indígena é chave para conduzir algumas análises. No entanto, os contextos sociais onde a identidade das pessoas indígenas se (re) produz foram alterados significativamente. Essas mudanças são produzidas pela assimilação ou integração das comunidades indígenas às culturas nacionais dominantes. Nesse contex- 
to, surgem limitações no uso dessa categoria, uma vez que a identidade indígena teria um componente político e legal relacionado com as necessidades dos governos. Portanto, uma reflexão crítica sobre o uso da identidade indígena é necessária nos enfoques epistemológicos e metodológicos de pesquisa. Este artigo argumenta que a pesquisa sobre os povos indígenas deveria avaliar como é feita a inclusão da identidade indígena, que é socialmente coproduzida na interação com o Estado e suas instituições. Desse modo, ela não necessariamente poderia constituir uma variável explicativa. Através da análise do discurso sobre pessoas e comunidades indígenas Aymara que surgiu na fronteira norte do Chile busco expor as lógicas utilizadas para definir a identidade. Concluise, então, que paulatinamente o processo de auto-identificação emerge em supostos indígenas constituídos e/ou reforçados pelas instituições, os quais deveriam ser revisados a partir de um enfoque descolonizado para inclui-los em pesquisas comparativas.

Palavras-chave: Indígenas, Identidade, Censo.

\section{INTRODUCCIÓN}

Este artículo explora aspectos de la identidad como una categoría de análisis en los estudios comparativos de poblaciones Indígenas y no Indígenas. Empleando el análisis de discurso destaco los cambios sociales e institucionales sobre la concepción de la identidad Indígena, para luego discutir su empleo metodológico en los estudios comparativos. Particularmente, en términos discursivos, la identidad es co-producida por los Pueblos Indígenas y las instituciones del Estado. Estas últimas ocupan un rol importante en la definición de atributos de estas identidades en los países que otorgan reconocimiento legal a sus Pueblos Indígenas. Para ejemplificar este proceso, se tomaron discursos puntuales acerca de las personas y organizaciones Indígenas localizadas en el norte de Chile, específicamente en la región de Arica y Parinacota. Esta región se halla en la frontera de Chile con Perú y Bolivia, lo cual otorga otras particularidades a tomar en cuenta en el presente caso. La evidencia está basada en documentos emitidos por autoridades y organizaciones Indígenas.

El análisis de discurso es una metodología interpretativa y explicativa, que toma citas en un contexto histórico e ideológico específico para describir la conexión entre el lenguaje y las relaciones de poder (1). Este método de investigación busca analizar las narrativas de personas respecto de una o múltiples realidades (2). En este caso, los discursos oficiales en torno a la indigenidad han sido fundamentales en la construcción de la identidad individual y colectiva de las personas Indígenas en un territorio. Particularmente, como más adelante se presenta, esto ocurre a partir del reconocimiento legal de los Pueblos Indígenas y el establecimiento de una estructura institucional de protección y desarrollo basada en la identidad Indígena. En este sentido, las identidades son discursivamente construidas en concordancia con la interpretación de memorias y experiencias (3). De esta forma, a medida que transcurre el tiempo las personas constantemente renuevan sus identidades en diver- sos contextos a través del acto de la narración. El lenguaje, entonces, es fundamental para construir identidades y establecer políticamente hechos particulares. Hansen afirma que la identidad es política, social y relacional (4). Por lo tanto, para analizar la identidad Indígena, en este caso la Aymara, es necesario entender los discursos que han emergido en la interacción con las autoridades gubernamentales.

\section{CONTEXTUALIZACIÓN}

Desde que los países han reconocido a los Pueblos Indígenas en sus respectivas legislaciones, investigadores, profesionales y políticos han estado preocupados no sólo en cuantificar y caracterizar la población Indígena, sino también determinar brechas en relación a la población no Indígena. Los gobiernos han asumido la responsabilidad de monitorear y cerrar estas divergencias, específicamente aquellas relacionadas con salud, educación y empleo. Por ejemplo, cada año el Primer Ministro de Australia es responsable de emitir un informe al Parlamento en el cual describe el cierre de brechas entre las poblaciones Aborigen e Isleña del Estrecho de Torres y no Indígena. Para los gobiernos es fundamental la producción de estadística que permita hacer más objetiva la realidad de la población Indígena y el impacto de las políticas públicas en el cierre de brechas. Los criterios, metodologías y resultados obtenidos por las instituciones oficiales sirven, en muchos casos, de referencia para los investigadores en sus estudios comparativos.

¿Quién es Indígena? Es una pregunta recurrente que los gobiernos enfrentan dada la heterogeneidad de los Pueblos Indígenas a través de las diferentes escalas territoriales y administrativas. Particularmente, las autoridades han usado diferentes criterios para contar la población Indígena, tal como la auto-identificación, ascendencia, costumbres, membresía, características físicas, entre otras (5). Entre ellas, la Organización de las $\mathrm{Na-}$ ciones Unidas y la Organización Internacional del Trabajo han recomendado usar la auto-identificación como criterio principal (6). Bajo este criterio las personas y comunidades determinan ellas mismas su propia identidad, la cual es construida en relación a la percepción de los "otros" y considerando las categorías territoriales y socio-económicas (7). Aunque este medio no puede revelar las múltiples identidades, varios países respetan el derecho de auto-identificación a todos quienes se reconozcan como parte de un Pueblo Indígena. Sea este u otro el criterio, Axelsson y Sköld advierten que los gobiernos utilizan una perspectiva colonial al interpretar la identidad Indígena desde un único criterio (8). Así, indirectamente, la auto-identificación promueve en las personas Indígenas la desconexión de sus territorios y lenguas ancestrales puesto que no son requisitos para lograr el reconocimiento Indígena. En esta condición, personas podrían declarar ser Indígenas solamente con el objetivo de acceder al esquema gubernamental de protección social sin considerar las necesidades del Pueblo Indígena al cual dicen estar adscritos. Aunque la auto-identificación es un criterio aceptado para cuantificar a la población Indígena, los gobiernos definen otros para establecer la identidad desde la cual puedan interactuar con las instituciones. 
En los países latinoamericanos, el proceso de asimilación (voluntaria o forzada) ha contribuido a rehacer varias de las características consideradas como tradiciones de la identidad Indígena. Los países, de una manera intencionada o no, han establecido mecanismos de asimilación con el objetivo de integrar a los individuos Indígenas en sus culturas nacionales. Consecuentemente, descendientes de los primeros Indígenas integrados podrían desarrollar una identidad que difiera de sus padres o abuelos. No obstante, la asimilación involucra la socialización política de los principios y valores nacionales, a través de la cual se acepta o refuta al gobierno. Corntassel afirma que la demanda de derechos especiales resulta en la politización de la identidad Indígena (9). En este contexto histórico, político y social, tanto gobiernos como investigadores requieren un mejor entendimiento de los procesos que explican la identidad de las personas Indígenas (8). Para profundizar en esta complejidad, este artículo presenta la experiencia de Chile respecto de las comunidades Indígenas existentes en la frontera norte del país.

La región de Arica y Parinacota, ubicada en el norte, estuvo bajo la soberanía de Perú hasta la Guerra del Pacífico que ocurrió entre 1879 y 1883 . Como resultado de este conflicto Chile ocupó las provincias peruanas de Tacna y Arica, que, a diferencia de otros territorios anexados, no contaba con depósitos de salitre. De acuerdo al Tratado de Ancón de 1883, después de 10 años de ocupación, las personas en estas provincias podrían decidir si ellas querían pertenecer a Chile o Perú. Debido a esta condición, Chile implementó un proceso de asimilación conocido como Chilenización, el cual tuvo como objetivo la población rural de la región que hoy día es reconocida como Indígena (10). En ese entonces, las autoridades percibían que las personas que vivían en los pueblos del altiplano de las provincias ocupadas estaban a favor de la nacionalidad peruana. Sin embargo, de forma alternativa al plebiscito y luego de varios años de negociación, las dos partes acordaron en el Tratado de Lima de 1929 que la provincia de Arica permanecía en Chile y la provincia de Tacna regresaba a Perú.

Dada la ausencia de políticas para mejorar las condiciones de vida en el altiplano, apareció una inequidad espacial entre Arica y los pueblos del altiplano, lo cual motivó la migración rural-urbana de las familias Indígenas (11). Junto con ello, el desarrollo urbano y económico de Arica también atrajo Indígenas desde otros países, particularmente desde Bolivia (12). Durante estos procesos sociales, la identidad Indígena no fue una categoría en las políticas públicas. Los gobiernos no promulgaron ninguna ley especial para las personas y comunidades Indígenas en general, como sí lo hicieron para algunas comunidades Mapuches (7). Esta situación cambió cuando el gobierno reconoció la pre-existencia de Pueblos Indígenas en su territorio.

El primer gobierno democrático, después de la dictadura militar (1973-1990), creó un esquema institucional para proteger y fomentar el desarrollo de los Pueblos Indígenas basado en el reconocimiento de su identidad. En 1989, Patricio Aylwin, candidato presidencial de una coalición de partidos políticos de centro-izquierda, acordó el apoyo de las organizaciones Indígenas en las elecciones presidenciales a cambio de la creación de una ley especial para ellos. Este pacto fue conocido como 'Acuerdo de Nueva Imperial', el cual fue firmado días antes de la elección y se tradujo en la Ley Indígena No. 19.253 en octubre de 1993. Sin embargo, aunque los dirigentes Indígenas habían acordado con el gobierno un reconocimiento completo como es recomendado por las organizaciones internacionales, el parlamento definió a los Pueblos Indígenas como Etnias Indígenas con el fin de reducir su estatus político (13). A pesar de esta limitación, las personas Indígenas y sus organizaciones comenzaron a interactuar con las instituciones del Estado a través de su identidad.

Al igual que otros países, Chile adoptó la auto-identificación como criterio principal (14). Uno de los primeros instrumentos en incorporar la identidad Indígena como categoría fue el censo nacional de 1992. Desde entonces, el formulario censal ha considerado una pregunta para que las personas se identifiquen como miembros de alguno de los Pueblos Indígenas reconocidos (Ver Tabla 1). Sin embargo, el método ha cambiado en consideración a los intereses del gobierno (14). Por ejemplo, el censo de 1992 presentó dos limitaciones; primero, hacía referencia al concepto 'cultura Indígena' sin considerar la noción de Pueblo Indígena, y segundo, la pregunta sólo podía ser respondida por mayores de 14 años que tuvieran la nacionalidad chilena. Es importante reconocer que en Chile habían personas nacidas en otros países que se identificaban con Pueblos Indígenas reconocidos por la legislación nacional.

Tabla 1: Preguntas en los censos chilenos para contar la población Indígena

\begin{tabular}{|c|l|}
\hline CENSO & \multicolumn{1}{c|}{ PREGUNTA } \\
\hline 1992 & $\begin{array}{l}\text { Si usted es chileno, ¿Se considera perteneciente a alguna de las siguientes culturas? Mapuche, Aymara, } \\
\text { Rapa Nui, o ninguna de las anteriores. }\end{array}$ \\
\hline 2002 & $\begin{array}{l}\text { ¿Pertenece usted a alguno de los siguientes pueblos originarios o Indígenas? Alacalufe (Kawashkar), } \\
\text { Atacameño, Aymara, Colla, Mapuche, Quechua, Rapa Nui, Yámana (Yagán), o ninguno de los anteriores. }\end{array}$ \\
\hline 2012 & $\begin{array}{l}\text { ¿Se considera perteneciente a algún pueblo Indígena u originario? Sí o No } \\
\text { [Sí] ¿A cuál? Mapuche, Aymara, Rapa Nui, Likan Antai, Quechua, Colla, Diaguita, Kawésqar, Yagán o Yámana } \\
\text { u otro (Especifique) }\end{array}$ \\
\hline
\end{tabular}


En Chile, el censo de 2002 es la fuente de información más reciente sobre los Pueblos Indígenas. Respecto del censo 2012 que fue desarrollado durante el gobierno de Sebastián Piñera, dos comisiones, una nacional y otra internacional, cuestionaron su metodología y resultados $(15,16)$. Por esta razón, el gobierno excluyó este censo para definir políticas públicas y fueron considerado como estadística no oficial. Entonces, de acuerdo al censo 2002, la población nacional Indígena fue de 692.192 personas, de las cuales 29.484 están en la región de Arica y Parinacota. Éstos representan el 4,3\% del total de la población Indígena. Respecto del Pueblo Aymara, tuvo una población nacional de $\mathbf{4 8 . 5 0 1}$ habitantes. $\mathbf{2 5 . 7 3 0}$ de ellos están localizados en la región de Arica y Parinacota $(53,1 \%$ de la población nacional Aymara) y de los cuales 23.288 están en la comuna de Arica (90,5\% de la población regional Aymara). En el caso del Pueblo Aymara existe una particularidad dado el importante aumento de su población en los últimos años, en contraste a la escasa población que existía antes del censo de 1992. De esta forma, aunque el criterio de auto-identificación no fue incluido en censos anteriores al de 1992, la población Indígena puede ser estimada a través de un criterio territorial. En la región de Arica y Parinacota, la población en las áreas reconocidas como Indígenas por el Estado no superó las 7.274 habitantes entre los censos de 1930 y 1989. A partir de este dato, no se puede decir que toda la población se auto-identificaba como Indígena, sino más bien representa un máximo. La diferencia entre esta cifra y la población que se ha auto-identificado como Aymara en los últimos censos sugiere que el incremento en la población Aymara local no puede ser explicado a través de factores demográficos. En este sentido, es probable que la interacción entre los descendientes de familias Indígenas nacionales y extranjeras, así como también los mecanismos de identificación, podrían haber incidido en los procesos de formación de la identidad Aymara a nivel local.

El censo ha permitido contar y localizar las personas Indígenas en el país. Sin embargo, el gobierno ha implementado dos mecanismos más para identificar a la población Indígena en Chile. Primero, para mejorar la caracterización social y económica de la población Indígena, la categoría Indígena fue incluida en la Encuesta de Caracterización Socioeconómica Nacional (CASEN) al igual que el censo. En particular, esta encuesta desde 1985 evalúa cada tres y cuatro años el impacto de los programas sociales, particularmente las fuentes y niveles de ingreso. La encuesta CASEN incluye una pregunta para identificar población Indígena desde 1996, que fue modificada en el 2006. Desde ese año, la pregunta considera como criterios la pertenencia a los Pueblos Indígenas y el reconocimiento de la ascendencia Indígena.

Segundo, el Estado definió un mecanismo de identificación basado en una lista de apellidos relacionados con cada Pueblo Indígena para categorizar la población. Todas las personas, o alguno de sus ascendientes hasta tres generaciones anteriores, que hayan tenido al menos un apellido Indígena obtendrán una acreditación oficial de parte del Estado. Las personas sin apellidos Indígenas tienen la alternativa de identificarse como Indígena presentando como argumento sus rasgos culturales asociados con algún Pueblo Indígena. El Estado, a través de la Corporación Nacional de Desarrollo Indígena (CONADI), entrega certificados que autentifican la identidad Indígena a aquellas personas que cumplan con estos requisitos. El certificado es requerido para otros procesos tales como la formación de organizaciones Indígenas, el acceso a beneficios sociales, la participación en concurso de proyectos, la votación en elecciones Indígenas, entre otros.

En resumen, el reconocimiento de los Pueblos Indígenas produjo no sólo uno, sino tres mecanismos de identificación basados en diferentes criterios o parcialmente similares (17). En este sentido es posible apreciar dos periodos, un antes y un después del reconocimiento legal de los Pueblos Indígenas a través de los cuales los discursos de identidad han cambiado. De esta forma, aunque en los análisis los Pueblos Indígenas se ven como una población homogénea a partir de su identidad, existen particularidades no tan sólo por Pueblo, sino también por comunidad Indígena. Como veremos en el siguiente análisis, la identidad del Pueblo Aymara en el norte de Chile ha sido co-producida en la interacción con las instituciones de gobierno.

\section{ANÁLISIS E INTERPRETACIÓN}

Para ilustrar cómo la identidad Indígena ha ido cambiando, se seleccionaron cuatro discursos que responden a momentos particulares de la historia local y emergen en la interacción entre personas Indígenas y autoridades. De esta forma, evidenciamos los dos periodos ya mencionados, y la construcción social e institucional de la identidad.

En el primer periodo, sin reconocimiento legal, el gobierno central no diferenciaba su política pública en relación a la identidad Indígena de su población. No obstante, en la región de Arica y Parinacota a finales de los años 50, de manera excepcional, el gobierno central crea una institución autónoma que asume, entre otras responsabilidades, el desarrollo de las comunidades localizadas en los pueblos del altiplano. Esta institución fue conocida como Junta de Adelanto de Arica (JAA). Ella fue la primera autoridad local en interactuar con las personas Indígenas enmarcada en las necesidades de desarrollo, inicialmente con apoyo de Naciones Unidas en un programa de asistencia técnica conocido como Plan Andino y luego de manera independiente. Esta institución adquiere relevancia particularmente después del golpe militar en 1973, puesto que la autoridad otorgó mayor importancia a la frontera y la seguridad nacional. Así, la dictadura militar estableció un órgano dentro de la JAA llamado Comité de Desarrollo del Interior (CDI) para reafirmar la soberanía territorial. Para implementar un plan, el CDI elaboró un reporte sobre las personas que habitan el altiplano. A pesar que la JAA trabajó con las comunidades Indígenas desde los años 60 , no se halla en los documentos una descripción oficial de la población Indígena hasta el momento que el CDI comienza a funcionar. En uno de sus documentos, el CDI caracteriza a la población del altiplano de la siguiente forma: 
"El elemento humano de nuestra región (área rural de Arica) se caracteriza por una estructura grupal más bien de carácter primitivo, con un cierto individualismo en su acción (poco espíritu comunitario), un cierto grado de desencanto que lo hace ser pasivo y una cierta lentitud en su reaccionar motivadas por un aislamiento de años y en un medio a menudo agresivo. Se puede agregar como una característica importante o destacable un cierto desarrollado espíritu migratorio, natural ante el atractivo de la ciudad. Su cultura se basa en valores de tipo profundamente rural (folk) que obviamente no coinciden con la vitalidad y las exigencias de los grupos urbanos, lo que por otra parte les ha mantenido un tanto ajenos a los avances tecnológicos de la época" (18).

Esquema para una programación de Desarrollo del Interior del Departamento Gerencia Técnica, Subgerencia de Estudios y Fomento, JAA. Marzo 1974

El desarrollo económico impulsado por la JAA empujó a una separación entre lo rural y urbano. En esta frontera, como indica la cita, la autoridad percibe una diferencia actitudinal en la población de los pueblos del altiplano. Desde esa percepción, las personas carecerían de una actividad colectiva entre ellas y en relación al medio ambiente contrario a lo que hoy se puede pensar. El desconocimiento de las prácticas culturales y organizativas de las comunidades probablemente se debió a que el altiplano era inaccesible y poco conocido. Las autoridades percibían que los individuos del altiplano no estaban preparados para la vida en la ciudad. Sin embargo, ellos observaron como positivo la necesidad de migrar. En aquel entonces, las personas Indígenas vieron la migración rural-urbana como una alternativa viable para mejorar sus condiciones de vida. En la ciudad, las personas Indígenas sin una identidad reconocida legalmente enfrentaron dificultades dadas las diferencias culturales entre la ciudad y los pueblos del altiplano. Durante esos años, la identidad Indígena podría haber sido una categoría que hubiera permitido diferenciar las condiciones de vida de las personas en la región y producir información específica de ellos. Asimismo, la identidad solamente era social y promovida a través de las familias.

La situación cambia a partir de 1993; el reconocimiento de la identidad produjo que personas Indígenas fueran nombradas autoridades y profesionales en la dirección local de CONADI. Desde estos roles, ellos promovieron en los pueblos del altiplano la adscripción a la identidad Indígena y la formación de organizaciones de acuerdo a la ley Indígena. De esta forma, converge la perspectiva institucional y la social respecto de la identidad Indígena. Durante el gobierno de Ricardo Lagos (2000-2006), se decidió elaborar un documento que recopilaría las historias y situación actual de los Pueblos Indígenas a lo largo de Chile. Para ello se conformó en el año 2001 un comité que estaría a cargo de la elaboración del documento, el cual requirió de la participación de personas Aymaras. Las oportunidades en que personas Aymaras pudieron presentar ante el comité fueron diversas a lo largo de todo el proceso. En una ocasión, como quedó registrado en las minutas, ellos tuvieron que explicar cómo su identidad se relacionaba con la nacionalidad, puesto que había inquietud respecto de las prácticas culturales asociadas a los países vecinos, Bolivia y Perú. De esta forma, uno de los representantes del Pueblo Aymara expuso:

"En primer lugar, definimos, [...], desde el punto de vista aymara, desde el punto de vista de la identidad, [...], respetamos la nacionalidad desde el punto de vista a qué país pertenecemos. Entonces habemos aymaras chilenos, aymaras bolivianos, aymaras argentinos, aymaras peruanos, e inclusive a lo mejor debemos haber aymaras norteamerican; desde el punto de vista jurídico, entonces no hay ningún problema. Pero si usted me dice chileno o no yo le digo sí, soy chileno y ahí está mi carnet (y) servicio militar y le puedo mostrar. Entonces, soy chileno desde ese punto de vista y administrativamente, obviamente soy chileno y estoy viviendo en este país y participo políticamente en este país y tengo aspiraciones digamos dentro de toda la estructura nacional, y hago valer mis derechos en ese sentido" (19).

Dirigente Aymara en la Comisión de Verdad Histórica y Nuevo Trato, 2001 sobre los problemas de las comunidades

A pesar que las personas Aymaras experimentaron un proceso de aculturación desde que fueron incorporados a Chile, las autoridades parecen dudar respecto a su integración en la sociedad nacional. En este sentido la cultura Aymara es transnacional, puesto que los estudios antropológicos y lingüísticos la localizan a través de los Andes en Bolivia, Perú, Chile y Argentina (ordenados por el tamaño de la población). En este contexto, el representante en aquella reunión enfatiza que las personas Aymaras son chilenas, no sólo porque poseen un documento que así lo indica, sino porque ellos cumplen con todos sus deberes cívicos. Particularmente, el servicio militar obligatorio hace referencia a poder formar parte de las fuerzas armadas, en otras palabras, proteger los intereses de Chile. De esta forma, no fue necesario que la ley mencionara que las identidades Indígenas están subordinadas a la nacional. En este caso han sido los propios Indígenas que han posicionado su identidad en ese nivel. Así, la identidad Indígena sólo puede ser entendida dentro del Estado que la reconoce. De esta forma, aunque se entienda que la identidad Aymara es transnacional, en la práctica hay diferencias significativas en su concepción, que también se puede dar en el resto de los países.

Entender la importancia de la estructura institucional para las personas Indígenas permite clarificar la emergencia de otros 
atributos asociados con la identidad. Uno de ellos es la variable de género. Mientras la mayoría de los dirigentes de organizaciones Aymaras eran hombres, la representación de la mujer Indígena en roles políticos era particularmente baja. Esta situación cambió debido a la incorporación de la equidad de género en las políticas públicas. Aunque la presidencia de Michelle Bachelet promovió la participación igualitaria del hombre y la mujer en el gobierno, fue el presidente Sebastián Piñera quien por primera vez designó una mujer en el cargo de directora de la oficina regional de CONADI en Arica y Parinacota. Después algunos meses en el puesto, algunos representantes Indígenas tuvieron un conflicto con la directora regional respecto de la asignación de los recursos. En solidaridad, una de las asociaciones de mujeres Indígenas existentes en ese momento emitió una declaración pública a la directora que explica cuáles han sido los avances durante su administración. Una parte de esta declaración mencionaba:

"Queremos resaltar y valorar que bajo su dirección se han abierto espacios donde las Indigenas de la zona podemos ser parte y trabajar en lo que atañe a nuestras actividades de participación, ya que anteriormente no fuimos escuchadas, ni menos convocadas a colaborar en los temas que nos conciernen. Sin embargo, actualmente se nos han generado ciertos espacios para capacitaciones económicas, políticas y sociales, así como la participación en la preparación del Congreso Internacional de la Mujer Indígena. También valoramos la voluntad de escuchar nuestros requerimientos, tal es así que por primera vez se concreta en esta administración, la creación de la Unidad de la Mujer Indígena, un espacio que hace mucho tiempo era demandado por las mujeres Indígenas de la región" (20).

\section{Coordinadora de Mujeres de Pueblos Origina- rios Arica y Parinacota en apoyo a la Directora de CONADI, 2011}

En la ciudad, la identidad Indígena representa una ventaja no sólo para acceder a beneficios sociales sino también para participar en espacios políticos. La identidad política es una fuente de legitimación, la cual no era accesible por las mujeres Indígenas. Como grupo social, ellas se han incorporado desde la perspectiva política en la sociedad Indígena. Así, los roles de género en las comunidades Indígenas están sometidas a extensivos cambios, los cuales significan nuevas formas de identificación y también de interacción con el gobierno. En el acto de emitir una declaración pública se puede apreciar el valor político de la identidad Indígena. Asimismo, se observa que los cambios en la estructura institucional y también la integración social, política y económica de la mujer Indígena han llevado a resignificar la identidad Indígena a nivel local. Como antecedente adicional, una vez que inicia la política de equidad de género, a nivel local aparecieron diferentes organizaciones, bajo la Ley Indígena, integradas solamente por mujeres Indígenas. No obstante, es importante notar que de esta forma se recrean las organizacio- nes en la ciudad, lo cual establece una distancia respecto de la realidad del altiplano.

Finalmente, el gobierno local adopta la identidad Indígena creando un discurso sobre la cultura contemporánea del Pueblo Aymara. Las autoridades incorporaron la identidad, prácticas y discursos asociados con el Pueblo Aymara en las actividades oficiales. Por ejemplo, en la inauguración de una infraestructura pública, un yatiri, quien es una persona Aymara que entre otras funciones ocupa el rol de líder espiritual, conduce una ceremonia ancestral Ilamada pawa. El yatiri pide permiso y éxito a la pachamama (conocida como Madre Tierra). En este sentido, las instituciones estratégicamente usan la identidad Indígena para socializar la política regional. En el año 2016, para promover una actividad de navidad financiada por el gobierno local, las autoridades elaboraron una narrativa para los medios de comunicación y redes sociales acerca de un niño Aymara y su experiencia en navidad. Lo siguiente es un fragmento de ese texto:

\section{"José Mamani, un niño aymara y su navidad en la ciudad de Arica}

José Mamani vive en el pueblo de Guallatire, comuna de Putre. A José le gusta ver su dibujo animado preferido, Peppa Pig. Ya es diciembre y, luego de terminar el año escolar, su familia decide pasar la navidad en Arica, viajando desde Guallatire hasta la ciudad en la camioneta de su papá. Cansado por el viaje, el niño José Mamani se duerme y comienza a soñar con su cerdita preferida. En sus sueños, José le cuenta a Peppa Pig que en noviembre de este año junto a su papá y otras personas, ayudaron a rescatar una ballena que había varado en la playa Arenillas Negras de Arica, internándola mar adentro. Además, le explica que pasará navidad en una gran fiesta junto a sus primos al pie del Morro" (21).

El gobierno local ha desarrollado su propia versión de la vida contemporánea de la población Indígena. En este sentido se enfatizan dos características: la conexión entre los pueblos del altiplano y Arica, y los apellidos Indígenas. Sin embargo, la narrativa se distancia de la realidad del Pueblo de Guallatire, puesto que actualmente se encuentra casi abandonado. Este sólo cuenta con presencia de empleados públicos pertenecientes a Carabineros y la Corporación Nacional Forestal. A pesar de esto, el gobierno local describe la vida de las personas Aymara de forma que puede resultar una realidad para quien no conoce la condición de Guallatire. Por lo tanto la identidad no sólo es ocupada estratégicamente por las personas Indígenas sino también por las autoridades. Sea por el interés de promover un evento de gobierno u otro, las acciones de las instituciones gubernamentales afectan la producción de la identidad, siendo en algunos casos más esencialista.

A través de estas cuatro narrativas asociadas a la identidad y prácticas de la población Aymara, es posible entender su 
producción en diversas realidades. Las citas presentadas son puntuales, pero permiten entender visiones respecto de la identidad de las personas Indígenas en la frontera desde ellas mismas y las autoridades locales. En este sentido es importante considerar dos efectos en la frontera: la migración rural-urbana y la constante nacionalización de la frontera, las cuales han sido transversales a los periodos de reconocimiento. La problematización ocurre en la forma de incorporar la identidad, que demuestra diferentes realidades, como una variable de categorización en los estudios comparativos de población en áreas de educación, salud, u otros.

\section{DISCUSIÓN}

La migración rural-urbana es uno de los fenómenos sociales que ha llevado a aumentar la relación entre las personas Indígenas y el Estado. En Chile, la migración Indígena rural-urbana es destacable en la región de Arica y Parinacota. De acuerdo al censo de 2002, la comuna de Arica concentra el 91,25 y el $90,5 \%$ de la población regional auto-identificada como Indígena y Aymara respectivamente (22). En la región se entiende que el territorio ancestral de los pueblos Indígenas se encuentra en el altiplano; por lo tanto, dada la migración hacia la ciudad se estima que en algunos casos se experimente una desconexión.

En este contexto, el gobierno juega un rol significativo en la promoción de la identidad Indígena contemporánea. Incluyendo la identidad Indígena en el censo nacional, el gobierno identificó las divisiones territoriales y administrativas con mayores poblaciones Indígenas. En este sentido, la estadística nacional y sub-nacional tiende a homogeneizar a las personas Indígenas. En otras palabras, la agregación de los datos individuales a diferentes niveles de análisis niega paulatinamente la realidad heterogénea. Como ya fue mencionado, Chile adoptó la auto-identificación como la base principal para identificar las personas Indígenas.

La auto-identificación no está exenta de limitaciones, debido a que los descendientes Indígenas pueden hacer y deshacer una identidad de acuerdo a las circunstancias. Como se presentó en los diferentes discursos, las personas Indígenas ubican su identidad respecto del contexto institucional. Específicamente, la auto-identificación "transforma el mecanismo de afiliación grupal en actos individualizados de elección psicológica y subjetiva" (14). Desde la experiencia de las comunidades Aymaras en el norte de Chile, la identidad articula patrones de interacción social que se han movidos desde una perspectiva cultural a otra institucional y/o política. Entonces, la identidad Indígena es más una variable socio-política que socio-cultural. Esta apreciación cabe en la emergencia de las mujeres Indígenas en el contexto político o cómo los representantes Indígenas posicionan su identidad respecto de la nacionalidad. Aun así, los estudios sobre personas Indígenas han incorporado la identidad como una forma de examinar disparidades que apuntan a enfatizar las razones culturales. Sin embargo, la identidad Indígena es frágil porque es estigmatizada por las personas no Indígenas. Así, la investigación sobre personas Indígenas puede reforzar ciertos estereotipos negativos (23). En consideración a las limitaciones, la discusión presenta dos métodos que ha sido ocupados para analizar a las personas Aymaras en la región de Arica y Parinacota.

En el primer caso, Caqueo-Urízar et al. crearon la Escala de Involucramiento en la Cultura Aymara (EICA) para medir el nivel de involucramiento de los niños en prácticas Aymaras (24). El EICA fue usado para cuantificar los síntomas de ansiedad y depresión en niños Aymaras y no Aymaras, a través del cual no fue posible identificar diferencias entre ambos grupos (25). Sin embargo, el trabajo concluye que el involucramiento significativo de los niños en la cultura Aymara constituye una protección a estos desórdenes mentales infantiles. Este instrumento emplea un enfoque de categorización externa basada en seis sub-escalas relacionadas con el uso de lengua en la familia, el uso personal del lenguaje, celebración de tradiciones, actividades tradicionales, danza y música. Sin embargo, más allá del uso de la identidad como variable explicativa, el cuestionario del EICA consulta sobre prácticas culturales que no son claras. Por ejemplo, la danza local es un sincretismo cultural reconocido como Aymara o Andino, también nacionalmente como Boliviano, Chileno o Peruano. En Arica, las personas identifican un conjunto de danzas locales (tarqueda, thinku, etc.), pero no hay un consenso sobre cuál de ellas es Aymara. En esta discusión, el thinku, por ejemplo, es una expresión reconocida como Aymara, pero también categorizada como boliviana (26). Además, las personas de los pueblos del altiplano indican que la danza en la cultura chilena Aymara es aquella que usa la música de la guitarra, mandolina o violín, y precisamente no corresponde al thinku o la tarqueda. Similares discusiones aparecen en el análisis de otras prácticas culturales incluidas en el EICA con excepción de la lengua Indígena. Además, es interesante entender que las personas Aymaras llegaron a Arica en diversas olas migratorias con una participación importante de Aymaras nacidos en otros países. Winker indica que las características sociales y ambientales pueden explicar de una mejor forma las diferencias entre migrantes y personas que crecen en un país identificado en la misma categoría étnica (27). Por lo tanto, la ausencia de diferencias entre niños Aymaras y no Aymaras podría tener una explicación en el grado de integración de la generación que tomaron parte en la investigación.

En otro caso, el Ministerio de Salud desarrolló otro método para identificar personas Indígenas basado en un grupo de criterios. Esta institución ha buscado mejorar la situación de salud de los Pueblos Indígenas en Chile. El problema principal para alcanzar este objetivo fue la escasez de información científica sobre las personas Indígenas. Entonces, el Ministerio asumió la responsabilidad de producir un reporte para cada Pueblo Indígena enmarcado en un proyecto epidemiológico socio-cultural. En Arica, este Ministerio preparó un informe sobre disparidades de salud para el Pueblo Aymara. La identificación Indígena fue basada en cuatro criterios: apellido familiar Indígena, relaciones familiares Indígenas, reconocimiento institucional y auto-identificación (28). Para aumentar la sensibilidad del análisis, el método apuntó a incluir la mayor cantidad posible de casos más que a refinar la población en estudio (29). En este sentido, una de las recomendaciones en la cuarta sesión del Foro Permanente sobre Temas Indígenas de la ONU en el 2005 
fue considerar múltiples criterios para capturar la identidad y condiciones socio-económicas (30). Por ejemplo, en Nueva Zelanda, el censo usa un criterio múltiple para identificar a la población Indígena que incluye la auto-identificación y el lenguaje (8). Sin embargo, un problema en los métodos de múltiple criterio son las fuentes de información que usan diferentes mecanismos para recopilar los datos. Por lo tanto, se requieren ajustes para calcular los indicadores de salud. En el caso de la investigación en salud, Kaplan y Bennett establecen que el análisis de diferencia étnicas debe considerar la limitación de los datos, la diferencia entre un factor de riesgo y marcador de riesgo basado en la categoría étnica y evitar la estigmatización relacionada con la identidad (31).

Para mejorar metodologías y métodos es necesario reconsiderar la participación de personas Indígenas, no solamente como sujetos de estudio. Los casos previos demuestran cómo los 'expertos' han desarrollado sus propios enfoques para incorporar la identidad de las personas Indígenas en sus estudios, en la mayoría de los casos ocupando fuentes desarrolladas por el gobierno. En este sentido, las personas Indígenas tienen algo que decir respecto de las estimaciones y disparidades que los expertos identifican. La participación de las personas Indígenas no puede estar limitada, sólo porque ellos viven en áreas urbanas, hayan perdido sus prácticas culturales o hablen una lengua no Indígena. Madden et al. menciona que estableciendo la colaboración entre las instituciones nacionales de estadística y los Pueblos Indígenas se contribuiría a alcanzar un comprensible rango de estadísticas (32). En este sentido, el establecimiento de una evaluación demográfica basada en las comunidades podría fortalecer la información. Una alternativa en este sentido es implementar un enfoque de colaboración enmarcado en la investigación participativa basada en la comunidad (CBRP en su sigla en inglés). Como hemos visto, después de la recolección de datos, el análisis es un proceso unilateral. El CBRP es un enfoque colaborativo y sistemático que apunta a crear conocimiento mutuo entre las comunidades e investigadores (33). De acuerdo a Rogers Stanton, éste contribuye a entender las dinámicas comunitarias y descolonizar la experiencia con las comunidades Indígenas (34). Sin embargo, Tuhiwai Smith indica que es necesario poner atención en la generación de conocimiento, porque podría afectar a las personas Indígenas (35).

La experiencia de la población Aymara en términos de producción de indicadores cuantitativos puede ser generalizada a otros Pueblos Indígenas y países. El Pueblo Aymara en Arica y Parinacota presenta un alto nivel de asimilación al contexto urbano. Los descendientes de los primeros migrantes Aymaras en el área urbana han adoptado las formas de vida contemporáneas. Esto significa que los Aymaras urbanos no son comparable con los Aymaras que todavía viven en las áreas rurales. En este sentido, priorizar la identidad podría hacer invisibles otras variables sociales, culturales y económicas. Así, disparidades en salud podrían ser analizadas ciegamente sin tomar en cuenta factores que emergen en otras inequidades. En este caso, la identidad Aymara e Indígena puede ser aglutinante de varias disparidades económicas y no económicas. Por ejemplo, Romero-Toledo y Castro establecen que las personas Aymara se localizan en la periferia empobrecida de Arica. Esto significa que ellos enfrentan una inequidad espacial a nivel micro (36). La situación es más compleja para mejorar el proceso de identificación. En Latinoamérica, los Estados crean espacios basados en la identidad a través de la legislación, los cuales los descendientes podrían ocupar o rechazar de acuerdo a su contexto social y económico. Esto representa una limitación que los investigadores y profesionales de ciencias sociales que reflexionan sobre el fenómeno Indígena deben superar metodológicamente, más que lograr un ajuste en el análisis de datos.

\section{CONCLUSIÓN}

La emergencia social, política y económica de las personas Indígenas a nivel nacional e internacional produjo la institucionalidad de la identidad y luego la producción de indicadores para identificar disparidades en diferentes áreas. La auto-identificación es el principal criterio usado por los gobiernos para contar la población Indígena; sin embargo, son empleadas otras metodologías de identificación para intereses complementarios (por ejemplo, la caracterización socio-económica). Ellas generan diferentes perspectivas para el desarrollo de estudios de comparación de indicadores en poblaciones Indígenas y no Indígenas. Los investigadores emplean tanto los marcos metodológicos y resultados oficiales con una escasa reflexión acerca de cómo la identidad Indígena es producida social e históricamente. El uso de un enfoque unilateral por los investigadores para estudiar la población Indígena sugiere la necesidad de descolonizar las prácticas de estudio y ajustarlas a las perspectivas de los Pueblos Indígenas.

\section{NOTAS}

Este trabajo fue presentado inicialmente en la Escuela de Verano sobre "Metodologías Cualitativas para la Transformación de la Frontera" organizado por la Universidad de Tarapacá en Arica, Chile entre el 12 y 13 de enero de 2017 . El proyecto de investigación al cual este artículo pertenece se encuentra en el Departamento de Español y Estudios Latinoamericanos de la Universidad de Sydney.

El autor es receptor de una beca del programa Becas Chile de CONICYT y contó con el apoyo adicional del Centro Interdisciplinario de Estudios Interculturales e Indígenas, subvención CONICYT/FONDAP/15110006, en el cual es investigador asociado en la línea de Investigación: Desarrollo y Medio Ambiente.

Aunque no existe una convención preferida u oficial en Latinoamérica, 'Indígena', 'Aymara' y 'Mapuche' están escritas con la inicial en mayúscula a través de este artículo porque están siendo usados como nombres propios; indican personas específicas quienes reclaman ser descendientes de los habitantes originales de sus tierras y no son migrantes de acuerdo a las fronteras post-coloniales de los Estados-nación. Así, reconozco la creciente identidad internacional y la diferencia política en sus propios contextos entre lo que es Indígena y no Indígena. 
Cómo citar este artículo: Choque Caseres, D. La identidad indígena interpretada como una categoría de análisis en los estudios de población. Enfermeria (Montev.). [Internet]. 2017 Oct [citado xxxxx]; 6 95-104. Disponible en: https:// doi.org/10.22235/ech.v6iEspecial.1457

\section{REFERENCIAS BIBLIOGRÁFICAS}

1. Jacobs K. Discourse Analysis and its Utility for Urban Policy Research. Urban Policy and Research. 2006;24(1):39-52.

2. Jäger S. Discourse and Knowledge: Theoretical and Methodological Aspects of A Critical Discourse and Dispositive Analysis. En: Wodak R, Meyer M, editores. Methods of Critical Discourse Analysis. 1ra. edición. London; Thousand Oaks,CA; New Delhi: SAGE Publications, Ltd; 2001. p. 3262.

3. Ovens A. Personal Politics and Identity in Student Teachers' Stories of Learning to Teach. En: Mattos AMA, editor. Narratives on Teaching and Teacher Education: An International Perspective. New York, NY: Palgrave Macmillan; 2009. p. 65-76.

4. Hansen L. Security as Practice: Discourse Analysis and the Bosnian War. New York, NY; London: Routledge; 2006.

5. Loveman M. National colors: Racial classification and the state in Latin America. New York, NY: Oxford University Press; 2014.

6. Short D, Lennox C. Handbook of Indigenous Peoples' Rights. Florence: Routledge; 2016.

7. Van Kessel J. Los aymaras bajo el régimen militar de Pinochet (1973-1990). En: Van Kessel J, editor. Holocausto al Progreso: Los Aymará de Tarapacá. 4ta edición. Iquique: Instituto para el Estudio de la Cultura y Tecnología Andina, IECTA; 2003. p. 259-300.

8. Axelsson P, Sköld P. Indigenous Peoples and Demography. The Complex Relation between Identity and Statistics. New York, NY: Berghahn Books; 2011.

9. Corntassel J. Who is indigenous? 'Peoplehood' and ethnonationalist approaches to rearticulating indigenous identity. Nationalism and Ethnic Politics. 2003;9(1):75-100.

10. Díaz Araya A. Aymaras, peruanos y chilenos en los Andes ariqueños: Resistencia y conflicto frente a la chilenización del norte de Chile. AIBR, Revista de Antropología Iberoamericana. 2006;1(2):296-310.

11. Van Kessel J. Los aymaras contemporáneos de Chile. En: Hidalgo L. J, Schiappacasse F. V, Niemeyer F. H, Aldunate Del S. C, Mege R. P, editores. Etnografía: Sociedades indígenas contemporáneas y su ideología. Santiago: Andrés Bello; 1996. p. 47-67.

12. Galdames Rosas L, Ruz Zagal R. La Junta de Adelanto de Arica y John V. Murra. Dos lecturas sobre el Desarrollo Andino en el Norte de Chile. Chungará: Revista de Antropología Chilena. 2010;42(1):257-70.

13. Gundermann K. H. Procesos étnicos y cultura en los Pueblos Indígenas de Chile. Alpha. 2013(36):93-108.
14. Angosto-Ferrández LF, Krodolfer S. Race, Ethnicity and National Censuses in Latin American States: Comparative Perspectives. En: Angosto-Ferrández LF, Krodolfer S, editores. Everlasting Countdowns : Race, Ethnicity and National Censuses in Latin American States. Newcastle: Cambridge Scholars Publishing; 2012. p. 1-40.

15. Bravo D, Larrañaga O, Millán I, Ruiz M, Zamorano F. Informe Final Comisión Externa Revisora del Censo 2012. Santiago: Instituto Nacional de Estadísticas; 2013.

16. Bianchini R, Feeney G, Singh R. Metodología de trabajo, conclusiones y recomendaciones del informa de la comisión internacional para el Censo de Población y Vivienda 2012. Santiago: Instituto Nacional de Estadísticas; 2013.

17. Gundermann K. H, Vergara del S. JI, Foerster G. R. Contar a los indígenas en Chile: Autoadscripción étnica en la experiencia censal de 1992 y 2002. Estudios Atacameños. 2005;30:91-113.

18. Comité de Desarrollo del Interior. Esquema para una programacion de Desarrollo del Interior del Departamento. Arica: Autor; 1974.

19. Gobierno de Chile. Informe Comisión Verdad Histórica y Nuevo Trato con los Pueblos Indígenas. Santiago: Autor; 2003.

20. Noriega Zegarra L. Mujeres indígenas apoyan gestión de Directora regional de CONADI. El Morrocotudo [Internet] 2011 Agosto 24 [Acceso el 1 Junio 2017]. Disponible en: http://www.elmorrocotudo.cl/noticia/sociedad/ mujeres-indigenas-apoyan-gestion-de-directora-regional-de-conadi.

21. Gobierno Regional de Arica y Parinacota. Globos gigantes con helio darán vida a Carnaval Navideño de Arica [Internet]. Arica:Autor; 2016 [Acceso el 1 Junio 2017]. Disponible en: https://gorearicayparinacota.cl/index.php/ noticias/564-globos-gigantes-con-helio-daran-vida-a-carnaval-navideno-de-arica.

22. Instituto Nacional de Estadísticas. Estadíticas Sociales de los Pueblos Indígenas en Chile - Censo 2002. Santiago: Autor; 2005.

23. Rivara FP, Finberg L. Use of the terms race and ethnicity. Archives of Pediatrics \& Adolescent Medicine. 2001;155(2):119.

24. Caqueo-Urízar A, De Munter K, Urzúa A, Saiz JL. Entre lo Aymara y lo Chileno: Escala de Involucramiento en la Cultura Aymara (EICA). Una aproximación interdisciplinar a la dimensión Aymara en la vivencia intercultural de estudiantes de enseñanza básica del norte de Chile. Chungará: Revista de Antropología Chilena. 2014;46(3):423-35.

25. Caqueo-Urízar A, Urzúa A, De Munter K. Mental health of indigenous school children in Northern Chile. BMC Psychiatry. 2014;14(1):11.

26. Droguett FF, Droguett RF. El tinku como expresión política: Contribuciones hacia una ciudadanía activista en Santiago de Chile. Psicoperspectivas. 2015;14(2):62-71.

27. Winker MA. Measuring race and ethnicity: Why and how? JAMA. 2004;292(13):1612-4. 
28. Ministerio de Salud. Situación de Salud de la Población Aymara en la región de Arica y Parinacota. Evidencias de inequidades étnicas en el norte de Chile. Arica: Autor; 2014.

29. Pedrero M-m, Oyarce AM. Una Metodología Innovadora para la caracterización de la situación de salud de las poblaciones indígenas de Chile: Limitaciones y potencialidades. En: Centro Latinoamericano y Caribeño de Demografía (CELADE), editor. Notas de Población Nro 89. Santiago: Publicaciones de las Naciones Unidas; 2009. p. 119-45.

30. Permanent Forum on Indigenous Issues. Report on the Fourth Session (16-27 May 2005). New York, NY: UN Economic and Social Council; 2005.

31. Kaplan JB, Bennett T. Use of race and ethnicity in biomedical publication. JAMA. 2003;289(20):2709-16.

32. Madden R, Axelsson P, Kukutai T, Griffiths K, Mienna
CS, Brown N. Statistics on Indigenous Peoples: Inter national Effort Needed. Statistical Journal of the IAOS. 2016;32(1):37-41.

33. Vukic A, Gregory D, Martin-Misener R, Vukic A, Gregory D, Martin-Misener R. Indigenous Health Research: Theoretical and Methodological Perspectives. Canadian Journal of Nursing Research. 2012;44(2):146-61.

34. Rogers Stanton C. Crossing Methodological Borders. Decolonizing Community-Based Participatory Research. Qualitative Inquiry. 2014;20(5):573-83.

35. Tuhiwai Smith L. Decolonizing Methodologies. Research and Indigenous Peoples. Second ed. New York, NY; London: Zed Books; 2012.

36. Romero-Toledo H, Castro F. Una Lectura actual de la geografía de los Aymara en el Norte Grande; 2017. 